\title{
Influence de la forme des attaches de sécurité sur leur comportement en service
}

\author{
Riadh Bahloul ${ }^{\mathrm{a}}$, Philippe Dal Santo et Alain Potiron \\ LPMI : Laboratoire Procédés, Matériaux et Instrumentation, Équipe E.R.T., École Nationale Supérieure d'Arts et Métiers CER \\ d'Angers, 2 Bd. du Ronceray, BP 3525, 49035 Angers Cedex, France
}

Reçu le 28 juin 2004, accepté le 19 décembre 2005

\begin{abstract}
Résumé - Les outils numériques actuels permettent de prendre en compte la majorité des paramètres prévisibles et quantifiables intervenant dans la conception, la fabrication et la mise au point d'un produit et permettent de mettre en place des modèles numériques tridimensionnels très précis. Ce travail présente une étude de faisabilité dans le cadre d'une démarche d'optimisation de forme des pièces de sécurité automobiles obtenues à partir de tôle à Haute Limite Élastique (H.L.E). La méthode proposée repose sur un algorithme d'optimisation utilisant des plans d'expériences. Elle permet de déterminer l'influence de la forme des pièces sur leur comportement statique en traction afin de simuler la résistance au choc de l'attache lors de son dépliage. La méthodologie des surfaces de réponse est utilisée dans un contexte d'optimisation pour l'exploitation des résultats. La technique numérique adoptée est basée sur la théorie de l'élastoplasticité couplée au dommage et avec écrouissage isotrope. La formulation de l'endommagement ductile isotrope de Lemaître utilisée permet une simulation fiable dans le cadre de la méthode des éléments finis à travers une étude paramétrique et comparative de trois formes géométriques d'attaches. Les calculs qui sont effectués sur le code ABAQUS/standard ont montré que le rayon du trou oblong permettant l'accrochage de la ceinture, est le paramètre géométrique qui a l'effet le plus prononcé sur les résultats de l'étude. L'analyse par surfaces de réponse montre que l'état de contrainte de von Mises n'évolue que très peu en fonction des autres variables considérées, mais par contre que les valeurs des contraintes sont beaucoup plus importantes quand le rayon du trou oblong augmente.
\end{abstract}

Mots clés : Optimisation de forme / endommagement de Lemaître / plans d'expériences / surfaces de réponse / essais dynamiques

\begin{abstract}
Influence of shape of safety fasteners on their mechanical behaviour. The current numerical tools make it possible to take into account majority of the foreseeable and quantifiable parameters intervening in the design, manufacture and the development of a product and allow to use very precise three-dimensional numerical models. This work presents a shape optimisation study of automotive safety parts obtained from sheet metal with High mechanical characteristics (High Strength Low Allowed steel H.S.L.A). The method suggested constitutes an optimisation algorithm using the experimental designs in order to determine the influence of the shape of parts on their static behaviour in traction making it possible to simulate the impact resistance of the fastener at the time of its unbending. The response surface methodology is used in a context of optimisation for the analysis of the results. This numerical technique is based on the theory of elastoplasticity integrating an isotropic hardening. The formulation of Lemaitre isotropic ductile damage is used and must ensure a reliable simulation within the framework of the finite element method. With a parametric and comparative study of three geometrical shapes, the results of the calculations are carried out by using the ABAQUS/standard code. They showed that the effect of the radius of oblong hole is the most significant geometrical parameter in this study. This was highlighted by the response surface method which shows that the state of von Mises stress does not evolve according to other variables considered; on the other hand the values prove much more significant influence when the radius of the oblong hole increases.
\end{abstract}

Key words: Shape optimisation / Lemaître's damage / experimental designs / response surface / dynamic tests

\footnotetext{
a Auteur correspondant :

Riadh.Bahloul@angers.ensam.fr
} 


\section{Introduction : Problématique générale et objectifs}

La recherche d'un gain de productivité accru, alliée à l'obligation de garantir la tenue en service des pièces mécaniques, nécessite la mise en place de procédures d'optimisation pour la conception de la majeure partie des composants issus de fabrication mécanique. Le contexte actuel en Recherche et Développement incite à alléger et à simplifier les composants tout en améliorant la fiabilité des produits et en diminuant leurs coûts. C'est un fait acquis depuis longtemps, qu'une pièce mécanique faisant partie d'une structure bien conçue se doit d'être optimisée. Cependant, l'implication dans le processus d'optimisation des contraintes de « marketing », de conception, de fabrication et de maintenance du produit, rend l'opération extrêmement complexe. Une approche simple du problème consiste alors à optimiser les différentes étapes du processus les unes après les autres, à l'aide de méthodes qui leur sont propres.

Certains procédés de travail des métaux sont très anciens mais demeurent en vogue pour la fabrication de pièces dites «standard ». C'est le cas des opérations liées au découpage et au pliage des ferrures d'ancrage en acier des ceintures de sécurité dont l'usage et la distribution sont devenus communs. Ces deux phases de mise en forme sont toujours présentes et s'enchaînent lors de l'élaboration de ces composants qui répondent à des normes de service très sévères. Ces pièces anciennes, apparues avec les premières ceintures statiques dans les années 60, parfois inchangées depuis, servent à fixer le brin mort de la ceinture sur la voiture. Ainsi se dessine la problématique complexe sur laquelle repose l'étude : il s'agit d'étudier l'influence de la forme des pièces de sécurité fabriquées en acier à haute limite d'élasticité (H.L.E) sur leur comportement en service en vue de leur résistance au choc et de les optimiser. L'objectif à long terme de cette étude est de prévoir la tenue mécanique d'une attache après sa mise en forme afin de maîtriser l'état de contrainte et d'endommagement du matériau qui influe sur la résistance aux chocs du produit fini. Cette recherche doit conduire à la levée de verrous technologiques associés à la faisabilité des pièces de sécurité et à leur tenue en service. Elle doit aussi permettre d'optimiser le composant en termes de fiabilité et de coût, ainsi que l'outillage mis en œuvre lors de la phase de fabrication. Pour cela, des techniques numériques utilisant par exemple la méthode des éléments finis, sont couplées à des algorithmes d'optimisation. La technique proposée est fondée sur des méthodes d'optimisation qui s'appuient sur des plans d'expériences [1] développés par Taguchi et sur l'utilisation de surfaces de réponse [2], méthodes qui peuvent être mises en ouvre pour améliorer la conception des pièces et permettre la maîtrise du procédé. L'objet de cette étude est d'appliquer ces techniques numériques à l'optimisation de la forme des attaches de ceinture.

Les objectifs poursuivis sont de deux ordres et concernent :
1. L'augmentation de la résistance des attaches en service.

Dans ce but, il faut minimiser les contraintes résiduelles induites par les modifications subies par le matériau lors de la fabrication de la pièce. En effet, les efforts mis en jeu lors de la mise en forme par déformations plastiques, soumettent le matériau à des sollicitations complexes qui modifient ses propriétés mécaniques et qui influent sur son comportement mécanique.

Cet objectif passe par une optimisation du procédé dont nous ne parlerons pas ici.

2. La minimisation du niveau de la contrainte de von Mises lors d'un choc appliqué à l'attache.

Cet objectif passe par une optimisation de la forme géométrique de l'attache en configuration plane.

Une sollicitation quasi-statique de traction uni-axiale remplacera l'action dynamique et l'optimisation est faite sur des attaches planes qui préfigurent les pièces réelles dépliées par le choc. L'étude est faite en fonction de critères géométriques qui ne sont pas toujours accessibles aux calculs analytiques.

\section{Caractérisation mécanique et rhéologique du matériau}

\subsection{Essai de traction}

C'est l'essai le plus courant, la forme des éprouvettes peut être normalisée (NF EN10002-1) et des conventions permettent de définir les différentes caractéristiques du matériau telles que $R e, R m, A \%, K, n \ldots$ Une étude expérimentale permet de déterminer les caractéristiques mécaniques et rhéologiques de l'acier H.L.E. S500MC [3]. La caractérisation du matériau a été effectuée par des essais de traction uni-axiaux sous chargement répété, à température et vitesse constantes. L'hypothèse d'endommagement faible est faite pour garder un module d'Young constant et ainsi retrouver la déformation plastique. La variation de la contrainte $\sigma$ en fonction de la déformation $\varepsilon$ met en évidence le durcissement du matériau et la figure 1 montre l'évolution de l'écrouissage sur la courbe de traction $[4,5]$. La valeur du module d'élasticité vierge $E_{0}$ mesuré est égale à $2 \times 10^{5} \mathrm{MPa}$ et le coefficient de Poisson $\nu$ a une valeur égale à 0,28 .

\subsection{Coefficients caractéristiques de la courbe d'écrouissage}

Le graphe de la figure 1 permet d'extraire la loi d'écrouissage $R\left(\varepsilon^{\mathrm{Pl}}\right)$ supposée isotrope en sollicitation uni-axiale sous la forme d'une fonction puissance :

$$
R\left(\varepsilon^{\mathrm{Pl}}\right)=K\left(\varepsilon^{\mathrm{Pl}}\right)^{n}
$$

$\left(\varepsilon^{\mathrm{Pl}}\right)$ est la déformation plastique cumulée. La déformation vraie est la déformation logarithmique définie par :

$$
\varepsilon=\ln (1+e) \text { avec } e=\frac{\Delta l}{l_{0}}
$$




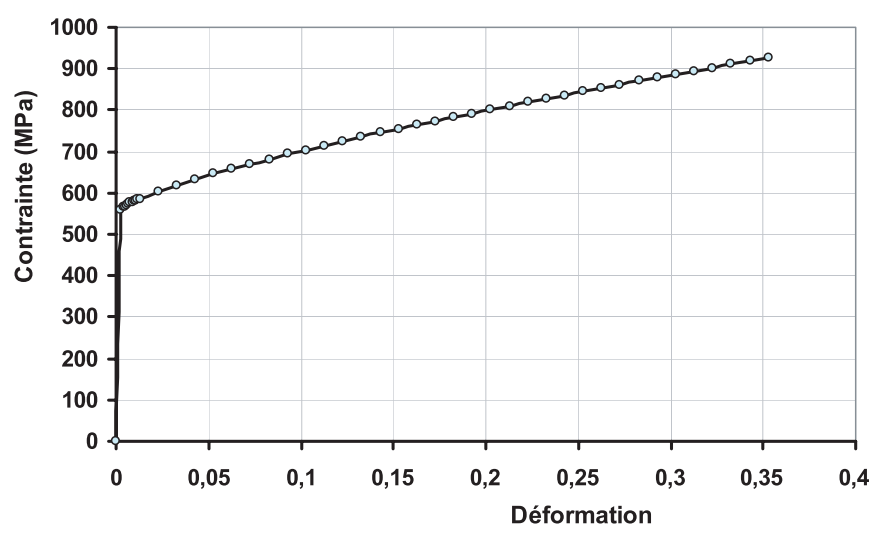

Fig. 1. Courbe de traction de l'acier S500MC.

Tableau 1. Caractéristiques de l'acier S500MC.

\begin{tabular}{lc}
\hline Limite élastique $\sigma_{y}$ & $560 \mathrm{MPa}$ \\
Résistance à la rupture $R m$ & $700 \mathrm{MPa}$ \\
Allongement après rupture & $23 \%$ \\
Lois d'écrouissage & $R\left(\varepsilon^{\mathrm{Pl}}\right)=800 \varepsilon^{0,745}$ \\
Dommage critique $D_{\mathrm{c}}$ & 0,21 \\
Déformation à la rupture $\varepsilon_{\mathrm{R}}$ & 0,35 \\
Déformation seuil d'endommagement $\varepsilon_{\mathrm{D}}$ & 0 \\
\hline
\end{tabular}

$\Delta l$ et $l_{0}$ désignent respectivement l'allongement courant et la longueur initiale de l'éprouvette de traction.

Sous forme logarithmique, l'expression (1) devient :

$$
\operatorname{Ln}\left(R\left(\varepsilon^{\mathrm{Pl}}\right)\right)=\operatorname{Ln}(K)+n \operatorname{Ln}\left(\varepsilon^{\mathrm{Pl}}\right)
$$

Il est possible par une régression linéaire, de déterminer les valeurs de l'exposant de la fonction d'écrouissage $n$ (pente de la droite) et de la consistance $K$ (ordonnée à l'origine) en traçant la courbe expérimentale $\operatorname{Ln}\left(R\left(\varepsilon^{\mathrm{Pl}}\right)\right)$.

Les valeurs ainsi identifiées sont $K=800 \mathrm{MPa}$ et $n=$ 0,745 .

L'hypothèse de décomposition additive de la déformation en une partie élastique $\varepsilon^{\text {el }}$ et une partie plastique $\varepsilon^{\mathrm{Pl}}$ est généralement adoptée et s'écrit alors :

$$
\varepsilon=\varepsilon^{\mathrm{el}}+\varepsilon^{\mathrm{Pl}}
$$

La loi de comportement de l'acier H.L.E. est alors la suivante :

Pour $\sigma \leq \sigma_{y}$ :

$$
\sigma=E \varepsilon^{\mathrm{el}}
$$

où $E$ représente le module d'Young initial $E_{0}$.

Pour $\sigma>\sigma_{y}$ :

$$
\sigma=\sigma_{y}+R\left(\varepsilon^{\mathrm{Pl}}\right)
$$

$\sigma_{y}$ est la limite élastique du matériau.

Le tableau 1 récapitule les caractéristiques du matériau.

\subsection{Couplage du modèle à l'endommagement}

La loi décrivant le comportement du matériau doit permettre la description des différentes étapes du processus de mise en forme des attaches, qui mettent en jeu des phénomènes d'endommagement et de rupture [6-8].

Dans ce cadre, il apparaît que la loi de comportement couplée à l'endommagement de Lemaître [9] est bien adaptée au matériau étudié. La fonction de charge est associée au critère de Von Mises, le calcul des contraintes et des déformations est basé sur une méthode de prédiction élastique accompagnée d'une correction plastique [10]. La matrice tangente a été calculée dans une procédure spécifique (routine utilisateur UMAT) qui permet d'implémenter des lois de comportement complexes dans le code de calcul Abaqus ${ }^{\circledR}$.

Cette approche a été utilisée et implémentée dans le code de calcul par éléments finis Abaqus Standard [11] pour l'intégration des équations constitutives nonlinéaires sous forme incrémentale. Dans de précédentes études [5] elle a montré sa robustesse et sa rapidité lors des simulations numériques.

Le taux de variation de la variable du dommage isotrope $D$ est défini par :

$\dot{D}=\frac{D_{\mathrm{c}}}{\varepsilon_{\mathrm{R}}-\varepsilon_{\mathrm{D}}}\left[\frac{2}{3}(1+\nu)+3(1-2 \nu)\left(\frac{\sigma_{H}}{\sigma_{\mathrm{eq}}}\right)^{2}\right]\left(\varepsilon_{\mathrm{eq}}\right)^{2 / n} \varepsilon_{\mathrm{eq}}^{\dot{.}}$

Ce modèle dépend de constantes intrinsèques à chaque matériau pour les propriétés du dommage. $\varepsilon_{\mathrm{D}}$ est la déformation seuil d'endommagement, en deçà de laquelle le dommage est nul ou négligeable. $\varepsilon_{\mathrm{R}}$ est la valeur de la déformation à la rupture pour laquelle le dommage a sa valeur critique $D_{\mathrm{c}}$ et $\varepsilon_{\text {eq }}$ représente la déformation plastique logarithmique équivalente.

L'identification de ce modèle consiste en une évaluation quantitative des trois coefficients caractéristiques $D_{\mathrm{c}}, \varepsilon_{\mathrm{R}}$ et $\varepsilon_{\mathrm{D}}$. La méthodologie employée s'explique par une mesure de pertes de module d'Young au moyen des essais de charge/décharge en traction unidimensionnelles [9]. Le tableau 1 récapitule les différents coefficients caractéristiques du dommage.

\section{3 Étude paramétrique}

L'objectif de cette étude est de déterminer l'influence de certains paramètres géométriques définissant la forme de l'attache, sur son comportement en service. Elle doit permettre de simuler la résistance au choc de l'attache après son dépliage. En effet, lors d'un choc, l'attache qui a été pliée se déplie de manière à se retrouver tendue dans une position quasi-plane. Dans la réalité bien que les opérations de pliage puis de dépliage entraînent des champs de contraintes et de déformation qui modifient le comportement de l'attache, nous ferons les hypothèses suivantes. 

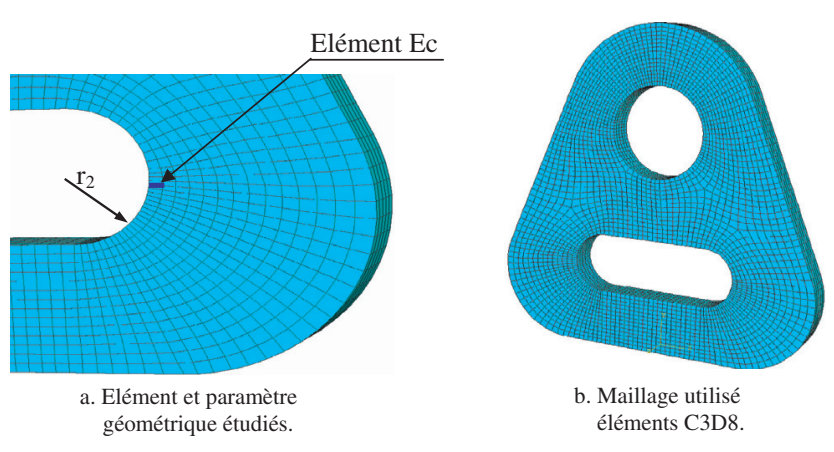

Fig. 2. Maillage utilisé et paramètre géométrique étudié.

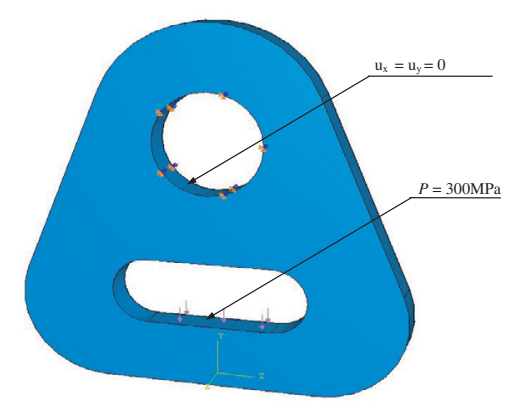

Fig. 3. Chargement et conditions aux limites.

\subsection{Hypothèses de l'étude}

On se place dans le cadre de l'étude du comportement de l'attache existante lorsqu'on modifie la forme du trou de passage de ceinture.

a. On suppose que la pièce est plane et exempte de tout champ de contraintes résiduelles.

b. La seule variable de conception est le rayon d'arrondi du trou oblong (Fig. 2a), qui reçoit la ceinture. Les valeurs varient de 3 à $6 \mathrm{~mm}$ avec un pas de $0,25 \mathrm{~mm}$. L'entr'axe des arrondis ne varie pas et la définition paramétrique de la géométrie actualise automatiquement la forme du trou.

c. L'action de la ceinture est assimilée à une pression uniforme qui s'exerce sur le bord inférieur du trou oblong. La traction sur l'attache est axiale suivant la direction $\vec{y}$ (Fig. 3). Les effets dynamiques sont ignorés.

d. La liaison de l'attache sur le châssis par boulon est modélisée par une condition d'encastrement sur l'alésage du trou de passage du boulon (Fig. 3).

\subsection{Modélisation de l'attache}

Les simulations numériques par éléments finis sont réalisées avec le code de calcul ABAQUS Standard. L'attache est modélisée comme un milieu continu tridimensionnel, avec un maillage obtenu par des éléments briques linéaires à 8 nœuds (C3D8) reporté sur la figure $2 \mathrm{~b}$.

Sur toute la surface interne de l'alésage (Fig. 3), les conditions aux limites suppriment les déplacements $u_{x}$ et

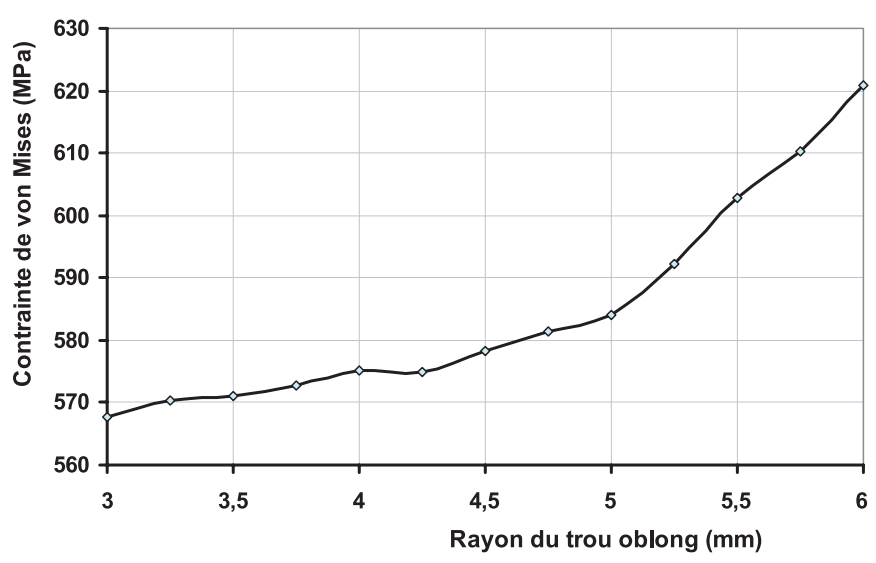

Fig. 4. Évolution de la contrainte de von Mises en fonction du rayon $r_{2}$ dans l'élément Ec.

$u_{y}$ suivant les axes $x$ et $y$. Les sollicitations de traction appliquées par la ceinture sur la pièce sont représentées par une pression suiveuse $P$ de $300 \mathrm{MPa}$ pour avoir une situation représentative du problème. Cette valeur entraîne de grandes déformations et des contraintes suffisamment élevées pour que certaines zones de la pièce soient plastifiées.

\subsection{Résultats}

Dans l'élément Ec qui se situe au milieu de l'arrondi du trou oblong, on étudie l'évolution de la contrainte de von Mises que l'on suppose être représentative du comportement sous charge. La figure 4 illustre la variation de cette contrainte en fonction du rayon $r_{2}$. On constate d'après le graphe une croissance de la valeur de la contrainte en fonction du rayon de l'arrondi du trou oblong. Cette évolution met en évidence les effets de concentration de contraintes dans les éléments adjacents aux arrondis.

Alors qu'on aurait pu s'attendre à une diminution de contrainte avec l'augmentation de l'arrondi, c'est le contraire qui se produit, ce qui peut s'expliquer par la diminution de la section de matière efficace avec l'augmentation du rayon.

En conclusion, dans les limites des variations imposées aux arrondis toutes dimensions égales par ailleurs, pour diminuer l'intensité des contraintes il faut diminuer la valeur du rayon d'arrondi.

Cette étude préliminaire ayant permis de quantifier la variation de contrainte avec le changement d'un seul paramètre géométrique, il paraît nécessaire d'élargir le champ d'investigation à d'autres paramètres qui peuvent piloter la forme de l'attache afin de mieux cerner le comportement et la résistance de la pièce.

De nombreuses études portant sur l'optimisation des formes de pièces chargées ont montré qu'il était souhaitable de canaliser les lignes de force (isovaleurs des contraintes principales) entre les zones d'application des chargements. On diminue ainsi les concentrations de contraintes, on favorise l'uniformité de la résistance locale 


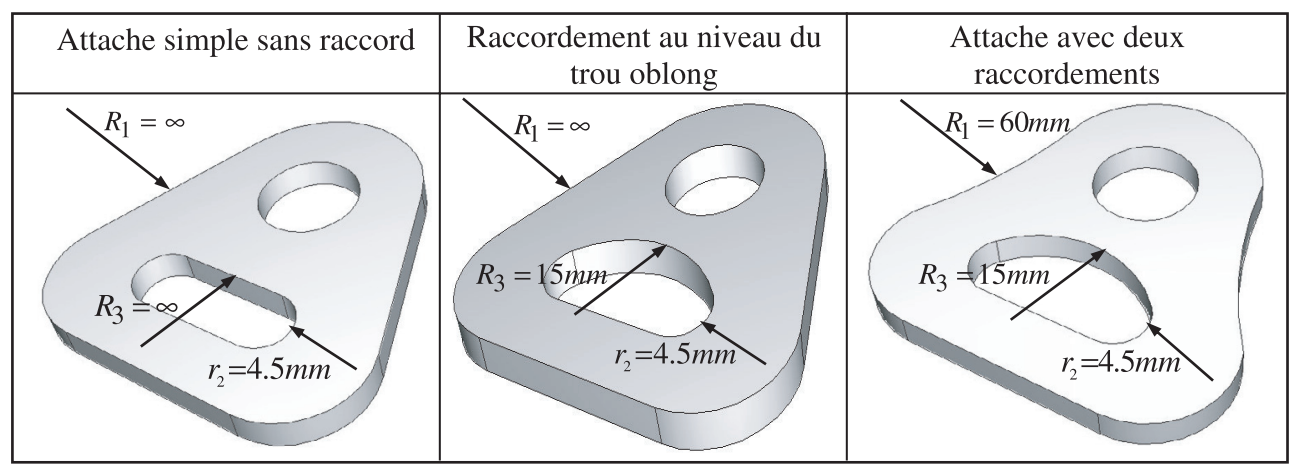

Fig. 5. Les trois formes géométriques de l'attache de ceinture.

Tableau 2. Contraintes maximales et dommage maximum.

\begin{tabular}{|c|c|c|c|c|c|c|c|c|c|c|c|c|}
\hline \multirow{2}{*}{ Résultats } & \multicolumn{2}{|c|}{$\begin{array}{l}\text { Contrainte } \\
\text { équivalente }\end{array}$} & \multicolumn{2}{|c|}{ Endommagement } & \multicolumn{4}{|c|}{ Contrainte de traction } & \multicolumn{4}{|c|}{ Contrainte de compression } \\
\hline & $\mathrm{N}^{\circ}$ élément & $\sigma_{\max }(\mathrm{MPa})$ & $\mathrm{N}^{\circ}$ élément & $D$ & $\mathrm{~N}^{\circ}$ élément & $\sigma_{11}(\mathrm{MPa})$ & $\mathrm{N}^{\circ}$ élément & $\sigma_{22}(\mathrm{MPa})$ & $\mathrm{N}^{\circ}$ élément & $\sigma_{11}(\mathrm{MPa})$ & $\mathrm{N}^{\circ}$ élément & $\sigma_{22}(\mathrm{MPa})$ \\
\hline $\begin{array}{c}1^{\mathrm{re}} \text { forme } \\
\text { géométrique }\end{array}$ & 8069 & 590,426 & 8069 & 0,0112 & 8415 & 599,834 & 7742 & 661,514 & 8406 & $-725,799$ & 7594 & $-414,794$ \\
\hline $\begin{array}{c}2^{\mathrm{e}} \text { forme } \\
\text { géométrique }\end{array}$ & 6680 & 591,877 & 6680 & 0,0119 & 7588 & 601,867 & 6811 & 665,790 & 7578 & $-726,216$ & 6617 & $-451,03$ \\
\hline $\begin{array}{c}3^{\mathrm{e}} \text { forme } \\
\text { géométrique }\end{array}$ & 6802 & 591,383 & 6390 & 0,0117 & 6979 & 602,838 & 5968 & 670,531 & 6971 & $-726,884$ & 6605 & $-454,561$ \\
\hline
\end{tabular}

ce qui entraîne généralement une diminution du poids de la matière. Ces quelques points conduisent à proposer certaines modifications de conception de forme des attaches.

\section{4 Étude comparative}

\subsection{Définition de l'étude}

Afin d'optimiser le comportement mécanique et la réponse des ferrures d'ancrage, on se propose d'étudier et de comparer les trois formes géométriques de la figure 5, en tenant compte de l'endommagement du matériau. Les conditions aux limites et le chargement sont identiques à ceux qui sont appliqués dans l'étude paramétrique précédente.

Les formes modifiées devraient amener un gain global de résistance comme nous l'avons déjà évoqué.

1. Une première simulation numérique a été réalisée sur la pièce initiale sans aucune modification géométrique afin de servir de référence.

2. La deuxième forme présente une modification du trou oblong. Un raccordement de rayon $R_{3}=15 \mathrm{~mm}$ entre les arrondis remplace le segment rectiligne. L'entr'axe est inchangé.

3. La troisième forme de l'attache correspond à une modification de la deuxième par un enlèvement de matière sur deux arêtes de la pièce formant deux arrondis de rayon $R_{1}$ égal à $60 \mathrm{~mm}$.

\subsection{Résultats obtenus}

Dans une première approche, chaque attache est sollicitée en traction en modifiant à chaque fois un paramètre géométrique. Pour faciliter leur interprétation, l'ensemble des résultats numériques concernant les contraintes maximales et l'endommagement maximum, est présenté dans le tableau 2. La numérotation des éléments correspondants est donnée sur la figure 6 .

Dans les trois cas de forme précédents, on constate que les valeurs maximales des contraintes équivalentes de von Mises et les valeurs d'endommagement sont presque égales et qu'elles sont localisées aux extrémités du trou oblong (Figs. 6 et 7). Dans cette région de l'attache, cela met en évidence l'influence du rayon $r_{2}$ de l'arrondi sur l'évolution de la contrainte maximale engendrée par une diminution de matière dans la section chargée. De même on observe sur les figures 7 que dans les zones proches du trou de fixation des attaches, les valeurs de contraintes et de dommage sont bien moins élevées qu'autour du trou de passage de ceinture. En effet, la condition d'encastrement sur la totalité du trou conduit à une grande raideur locale de la structure et limite les déformations et l'endommagement dans cette zone. Il peut être alors précisé que les écarts entre les résultats pour les différentes formes ne sont pas significatifs, ne serait-ce qu'en comparaison de la précision de la discrétisation due aux éléments finis. Cette remarque justifie a posteriori le choix des conditions d'encastrement au trou de boulon.

Si on observe les résultats du calcul dans les éléments situés sur l'axe de symétrie $Y$, peu de changements sont apparents.

\section{Contraintes $\sigma_{11}$}

Près du bord inférieur de l'attache, une légère différence (de 1 à $3 \mathrm{MPa}$ ) est constatée dans les valeurs des contraintes $\sigma_{11}$ correspondant à une traction suivant $X$. 


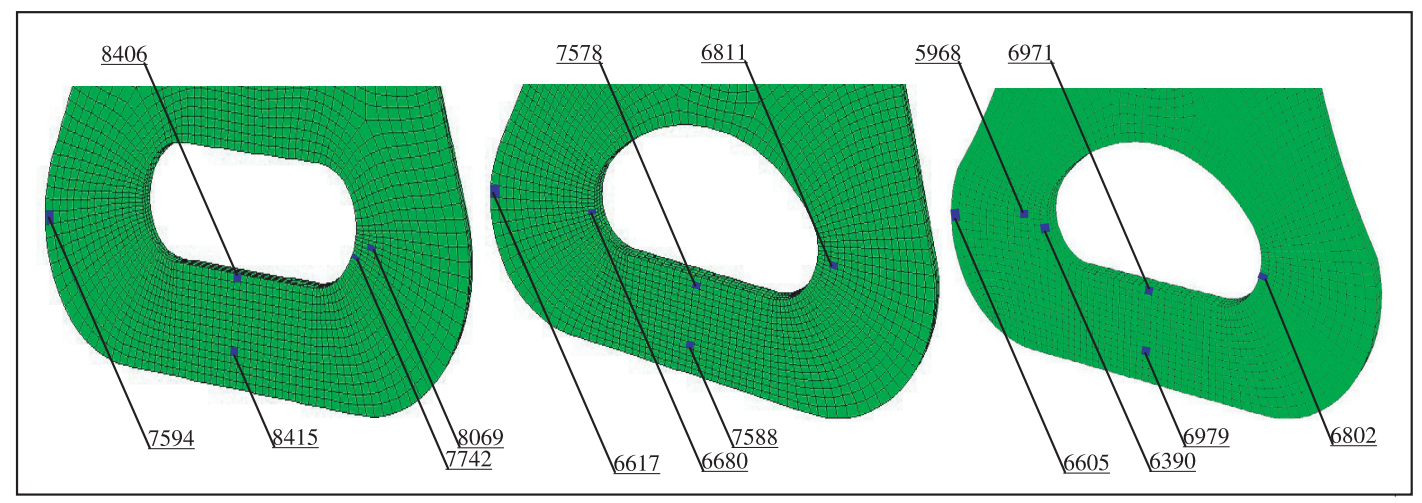

Fig. 6. Désignation des numéros des éléments.

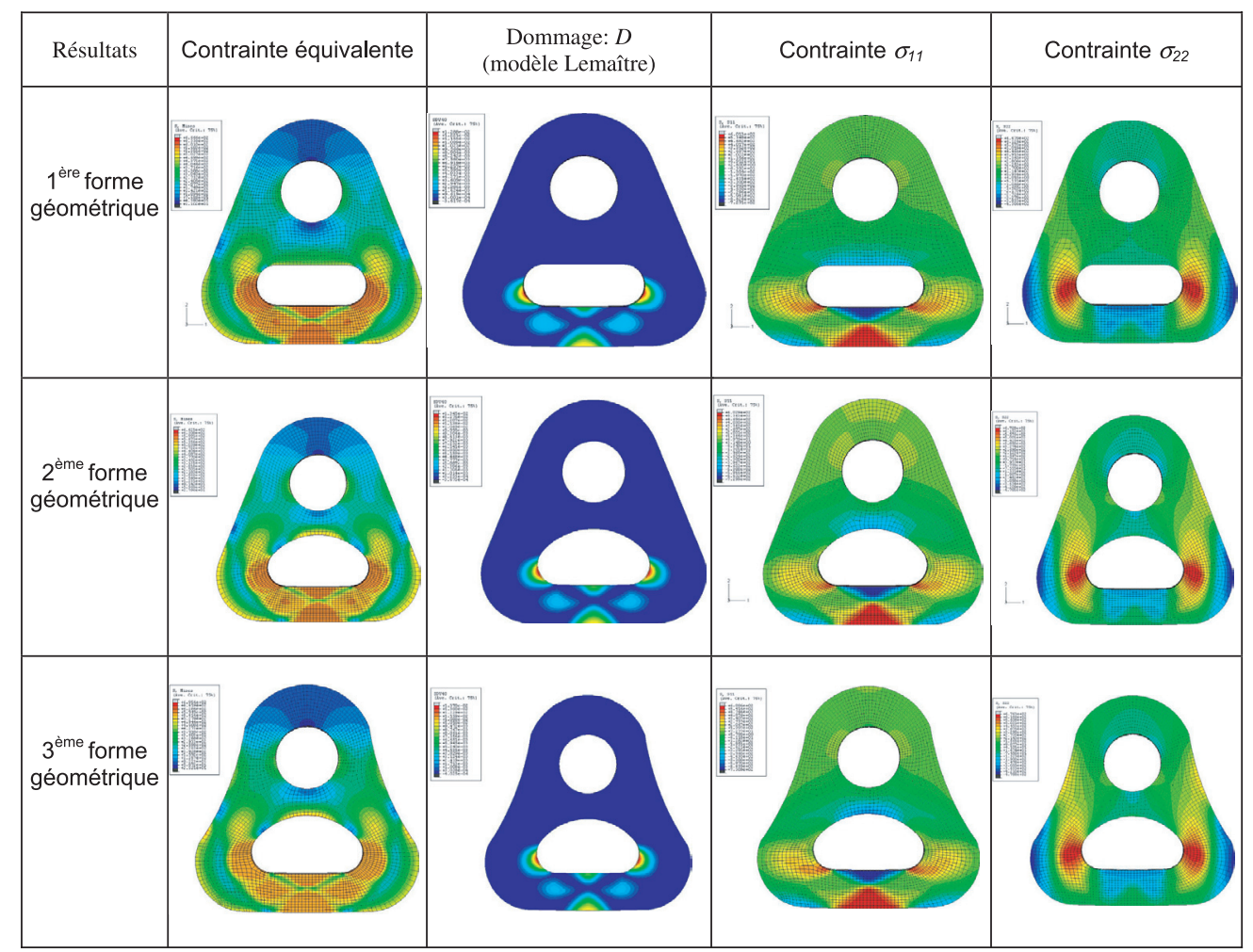

Fig. 7. Isovaleurs de distribution des contraintes et de l'endommagement.

Près du bord inférieur du trou oblong, l'état de contrainte de compression est peu influencé par les modifications de forme. Les éléments correspondants sont situés sur une même verticale portée par l'axe de symétrie de l'attache (Fig. 6).

La distribution de ces contraintes correspond grossièrement à une flexion plastique d'axe $Z$. Les valeurs des contraintes obtenues dépassent la limite élastique qui est de $560 \mathrm{MPa}$. Les valeurs maximales obtenues varient de 599,8 $\mathrm{MPa}$ pour la première forme à $602,8 \mathrm{MPa}$ pour la troisième en passant par 601,8 MPa pour la deuxième. Comme on le constate, ces valeurs sont très peu différentes les unes des autres et le gain obtenu en choisissant l'une ou l'autre des trois formes ne peut être que sur le poids qui est minimal avec la troisième.

\section{Contraintes $\sigma_{22}$}

Sur une ligne horizontale passant par les centres des demi-cercles de l'arrondi, les variations de $\sigma_{22}$ ne dépassent pas $10 \mathrm{MPa}$. Les valeurs positives et négatives peuvent s'expliquer par une flexion et une traction engendrées par la pression appliquée.

En ce qui concerne le dommage $D$, comme on peut s'y attendre il est concentré sur le bord des arrondis, là où les contraintes et les déformations plastiques sont maximales. Parmi les trois formes, il semble que la première entraîne un dommage minimal vraisemblablement relié à la plus faible valeur des contraintes obtenue pour cette forme.

L'étude précédente s'intéressait à l'analyse de l'influence des trois configurations géométriques sur les 
champs de contraintes et de dommage en vue d'une optimisation de l'attache.

L'objectif de la méthode d'optimisation développée dans la suite est d'avoir accès sans effectuer de simulation supplémentaire, à une estimation des niveaux de valeurs des différents champs pour toutes les combinaisons des paramètres qui agissent sur la forme. C'est pour cette raison qu'on a adopté une méthode de plans d'expériences et l'utilisation de surfaces de réponse [12] pour identifier les paramètres significatifs du problème d'optimisation.

\section{Plan d'expériences}

\subsection{Intérêt d'un plan d'expérience}

Les plans d'expériences ont un réel intérêt dans la résolution de nombreux problèmes industriels en évitant le coût élevé de nombreux essais tout en faisant gagner du temps. En particulier, ils sont rapides et faciles à mettre en œuvre puisqu'il s'agit simplement de caractériser l'influence des paramètres et des critères de conception sur les objectifs de l'étude concernée. De plus les méthodes mises en œuvre sont suffisamment génériques pour être utilisées dans des domaines très variés, tels que la mécanique, la robotique, le marketing, la gestion de production, etc. Ils sont relativement fiables sur des ensembles ou des systèmes non aléatoires. Afin d'optimiser l'organisation des expériences et surtout de les exploiter efficacement, nous avons retenu la méthode de Taguchi qui est basée sur des considérations rationnelles ainsi que sur des règles statistiques précises $[13,14]$. Le principe de cette méthode consiste à ne pas prendre en compte toutes les configurations, mais seulement certaines choisies pour leurs propriétés d'orthogonalité. Cette méthode permet d'établir un plan d'expérimentation comportant le minimum d'expériences compte tenu des résultats recherchés tout en apportant le maximum de précision.

Pour un problème complexe, de multiples paramètres sont susceptibles d'influer sur la réponse du système à étudier. La performance qu'on cherche à atteindre est caractérisée par une ou plusieurs réponses et on peut se demander quels sont les paramètres responsables des variations observées?

Ce type de problème peut être schématisé par une boîte noire (Fig. 8).

\subsection{Mise en place d'un plan d'expériences}

Dans la mise en œuvre par la méthode de Taguchi, appliquée à notre problématique, les paramètres utilisés sont purement géométriques. Ce sont les rayons $R_{1}, r_{2}$ et $R_{3}$ qui varient dans des intervalles bien définis (Fig. 9). Le plan factoriel complet qui a été mis en place, comporte 3 facteurs à 2 niveaux chacun. Ce plan représenté sous forme d'une matrice de 8 expériences, permet de faire toutes les combinaisons possibles entre les 3 facteurs. Le tableau 3 donne le domaine de variation des différents paramètres dont les limites sont illustrées sur la figure 9 .

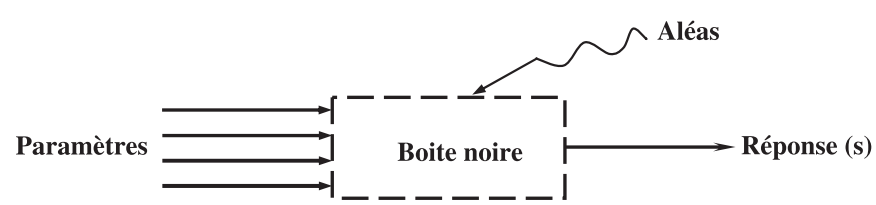

Fig. 8. Schéma du process.

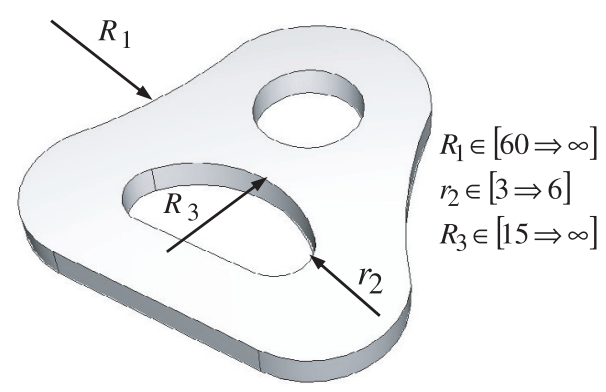

Fig. 9. Représentation des paramètres géométriques.

Tableau 3. Domaine de variation des paramètres d'entrée du plan d'expériences.

\begin{tabular}{ccc}
\hline Niveau & -1 & 1 \\
\hline Facteurs & & \\
$R_{1}$ & $\infty$ & $60 \mathrm{~mm}$ \\
$r_{2}$ & $3 \mathrm{~mm}$ & $6 \mathrm{~mm}$ \\
$R_{3}$ & $\infty$ & $15 \mathrm{~mm}$ \\
\hline
\end{tabular}

Tableau 4. Matrice d'expériences.

\begin{tabular}{ccccc}
\hline & & & \multicolumn{3}{c}{$\begin{array}{c}\text { Contrainte maximale } \\
\text { Essai } \mathrm{N}^{\circ}\end{array}$} & $R_{1}$ & $r_{2}$ & $R_{3}$ & 588 \\
\hline 1 & -1 & -1 & -1 & 586 \\
2 & -1 & -1 & 1 & 806 \\
3 & -1 & 1 & -1 & 803 \\
4 & -1 & 1 & 1 & 585 \\
5 & 1 & -1 & -1 & 587 \\
6 & 1 & -1 & 1 & 803 \\
7 & 1 & 1 & -1 & 798 \\
8 & 1 & 1 & 1 & \\
\hline
\end{tabular}

La réponse qu'on analyse est le maximum atteint par la contrainte de von Mises lorsqu'on fait varier les paramètres de l'étude.

Ces résultats qui correspondent à des « expériences virtuelles » sont déterminés par une simulation par éléments finis dans les mêmes conditions que précédemment. Le tableau 4 récapitule les valeurs des réponses trouvées sous forme d'une matrice d'expériences. Il servira comme base de données numériques pour l'analyse des résultats qui suit, à l'aide de surfaces de réponses.

\section{Application des surfaces de réponse}

L'analyse des réponses aux différents essais numériques est :

1. soit qualitative : étude de «screening » ou détermination des facteurs influents. 
Tableau 5. Identification des différents coefficients de la fonction objective.

\begin{tabular}{ccccccccccc}
\hline Coefficients & $\alpha_{0}$ & $\alpha_{1}$ & $\alpha_{2}$ & $\alpha_{3}$ & $\alpha_{11}$ & $\alpha_{22}$ & $\alpha_{33}$ & $\alpha_{12}$ & $\alpha_{23}$ & $\alpha_{13}$ \\
\hline Valeurs & 1,398 & $-0,003$ & 0,949 & $-0,001$ & $-0,003$ & 0,359 & $-0,0014$ & 0 & $-0,002$ & 0,011 \\
Coefficients & $\beta_{11}$ & $\beta_{22}$ & $\beta_{33}$ & $\beta_{12}$ & $\beta_{13}$ & $\beta_{21}$ & $\beta_{23}$ & $\beta_{31}$ & $\beta_{32}$ & $\gamma$ \\
Valeurs & $-0,003$ & $-0,02$ & $-0,0014$ & $-0,0009$ & 0,0111 & 0,00126 & $-0,00195$ & 0,0112 & $-0,00187$ & $-0,04$ \\
\hline
\end{tabular}

2. soit quantitative : méthodologie des surfaces de réponse, (variation des réponses en fonction des facteurs influents).

Dans tous les cas, elle a pour but de déterminer des modèles mathématiques approchés qui permettent d'exprimer les réponses en fonction des variables de l'étude $[15,16]$ (ici les paramètres géométriques). Ces modélisations sont déduites des valeurs issues des séries de simulations réalisées pour la constitution des plans d'expériences.

\subsection{Détermination de la fonction objective}

La fonction objective que nous avons choisie pour cette étude est la contrainte maximale de von Mises atteinte en fin de traction. Les trois paramètres de conception sont des paramètres géométriques correspondant aux trois rayons $R_{1}, r_{2}$ et $R_{3}$. Pour éviter une trop grande influence de l'un d'entre eux sur le résultat, ils sont normalisés. La fonction objective est rapportée à la pression $P$ appliquée par la ceinture sur l'attache.

Les valeurs normalisées qui ont été utilisées sont définies ci-dessous :

$$
\tilde{\sigma}_{v M}=\frac{\sigma_{v M}}{p} ; \rho_{1}=\frac{60}{R_{1}} ; \rho_{2}=\frac{3}{r_{2}} ; \rho_{3}=\frac{15}{R_{3}}
$$

avec :

$$
R_{1} \in[60, \infty], r_{2} \in[3,6], R_{3} \in[15, \infty]
$$

Les plages de variations des paramètres adimensionnels sont ainsi :

$$
0 \leq \rho_{1} \leq 1, \quad 0,5 \leq \rho_{2} \leq 1, \quad 0 \leq \rho_{3} \leq 1
$$

Dans la suite l'approximation de la fonction objective est définie par une fonction polynomiale cubique $f$ écrite sous la forme suivante :

$$
\begin{aligned}
f=\tilde{\sigma}_{v M_{\max }}=\alpha_{0}+\sum_{i=1}^{n} \alpha_{i} \rho_{i}+\sum_{i=1}^{n} \alpha_{i i} \rho_{i}^{2}+\sum_{i<j}^{n} \alpha_{i j} \rho_{i} \rho_{j} \\
+\sum_{i=1}^{n} \beta_{i i} \rho_{i}^{3}+\sum_{i=1, j=1, i \neq j}^{n} \beta_{i j} \rho_{i}^{2} \rho_{j}+\prod_{i=1}^{n} \gamma \rho_{i}
\end{aligned}
$$

Elle permet de représenter les valeurs des contraintes normalisées en fonction des rayons normalisés. Les coefficients $\alpha_{i j}, \beta_{i j}$, et $\gamma$ du polynôme sont déterminés par la minimisation de la somme des erreurs quadratiques $E^{\text {Quad }}$ par rapport à $\alpha, \beta$ et $\gamma$, à l'aide du solveur Mathematica [17].
$E^{\text {Quad }}$ est donnée par l'expression suivante :

$$
E^{\text {Quad }}=\sum_{i=1}^{N}\left(\sigma_{i}^{\text {simulé }}-f_{i}\right)^{2}
$$

$\sigma_{i}^{\text {simulé }}$ et $f_{i}$ sont respectivement les valeurs fournies par le calcul éléments finis et la valeur de la fonction $f$ pour un jeu de valeurs des paramètres.

Le tableau 5 récapitule les valeurs des différents coefficients de la fonction objective qui ont été calculés.

\subsection{Interprétation des résultats par surfaces de réponse}

Pour bien analyser et comparer les résultats en vue de déterminer les facteurs significatifs du problème d'optimisation, on donne à un paramètre une de ses valeurs limites et on trace la surface de réponse en fonction des variations des deux autres paramètres. La mise en œuvre a été faite à l'aide du logiciel Matlab [18].

Les évolutions de la contrainte maximale de von Mises sont illustrées sous forme de surfaces sur les figures 10a et $10 \mathrm{~b}$. Elles correspondent respectivement aux valeurs des rayons normalisés $\rho_{1}=0$ et $\rho_{1}=1$ et on peut voir qu'elles sont presque identiques, ce qui montre que ce paramètre a peu d'influence sur la distribution de la contrainte $\sigma_{v M}$. Par contre le gain de poids correspondant à $R_{1}$ maximum peut être intéressant. En ce qui concerne le rayon $R_{2}$ du trou oblong, il faut noter que le paramètre $\rho_{2}$ est un facteur essentiel dans la conception de l'attache puisque la variation de la contrainte qu'il entraîne est très importante.

Les figures 11a et 11b présentent les surfaces de réponse dans les deux cas où le rayon $\rho_{2}$ du trou oblong prend ses valeurs inférieure et supérieure. Les graphes montrent que l'évolution de la contrainte en fonction de $\rho_{1}$ et $\rho_{3}$ est croissante en fonction de $\rho_{2}$. Ceci parait physiquement logique puisque la section active de matière à l'extrémité du trou oblong diminue, entraînant un phénomène de concentration de contraintes.

Les surfaces de réponse données figures $12 \mathrm{a}$ et $12 \mathrm{~b}$ illustrent la réponse mécanique de la pièce de sécurité en fonction des rayons $\rho_{1}$ et $\rho_{2}$ quand on donne au paramètre $\rho_{3}$ les valeurs correspondant aux bornes inférieure et supérieure de son intervalle de variation. L'allure générale des deux surfaces est quasi plane mais les amplitudes des contraintes croissent fortement en fonction du rayon $\rho_{2}$, ce qui souligne une fois de plus l'importance de ce paramètre de conception. Comme a été mentionnée la faible 

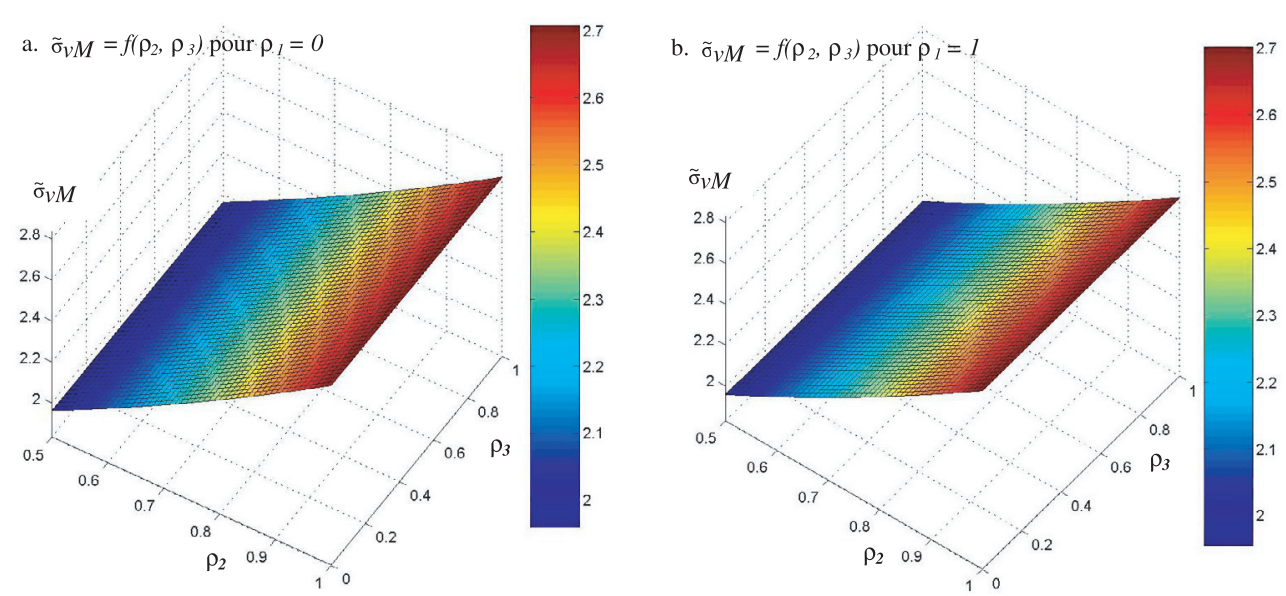

Fig. 10. Surfaces de réponse obtenues dans le cas $\rho_{1}$ fixé.
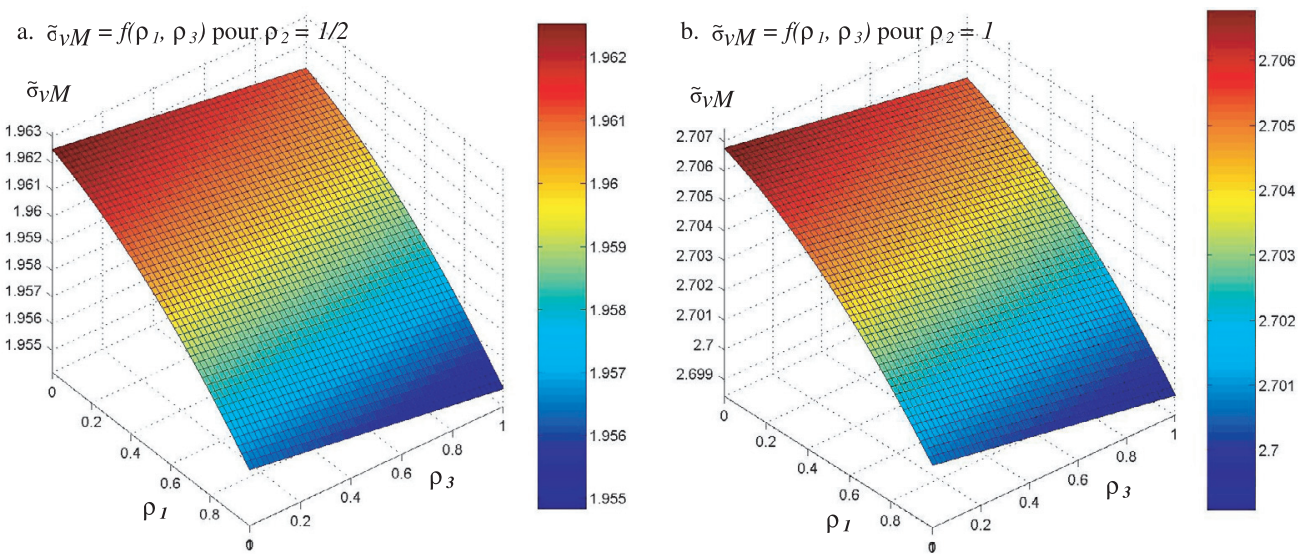

Fig. 11. Surfaces de réponse obtenues dans le cas $\rho_{2}$ fixé.
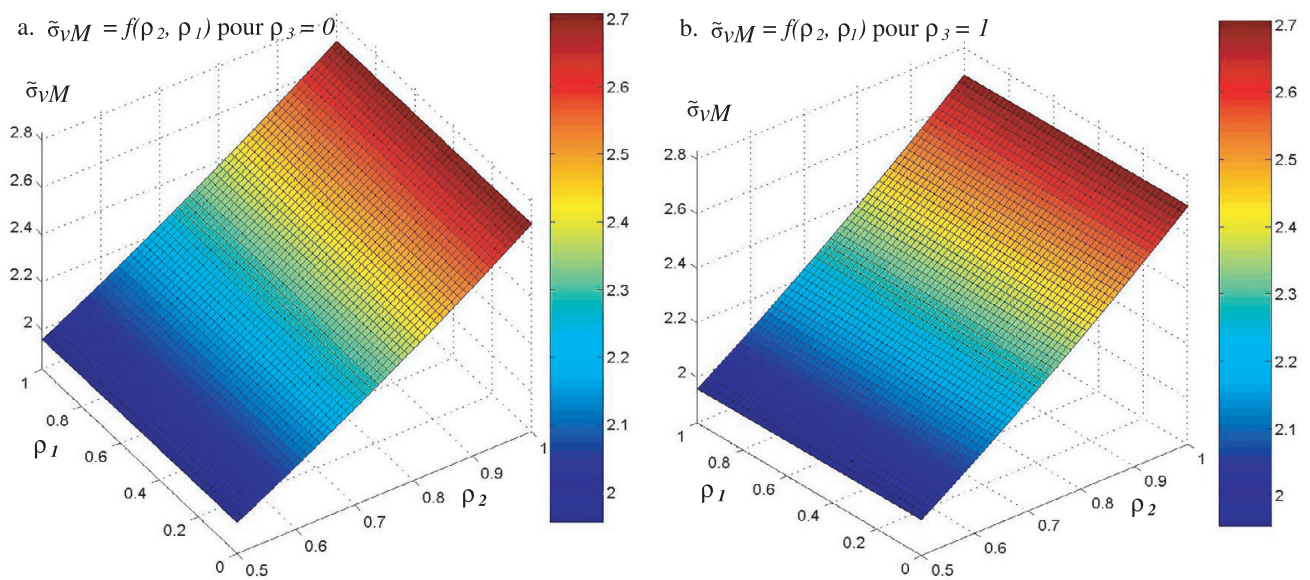

Fig. 12. Surfaces de réponse obtenues dans le cas $\rho_{3}$ fixé.

influence du rayon $\rho_{1}$, on pourrait aussi remarquer d'après la figure 12 la faible influence de $\rho_{3}$ sur l'évolution de la contrainte équivalente maximale puisque les deux surfaces présentent des allures presque identiques.

L'observation des deux graphes précédents ne fait pas apparaître d'influence significative du rayon $\rho_{1}$. En fait, pour un niveau quelconque du rayon $\rho_{2}$, la valeur de la contrainte reste invariable quel que soit la valeur de $\rho_{1}$.

\section{Conclusions}

Cette étude a eu pour but d'appliquer une approche simplifiée en vue d'analyser le comportement en traction de ferrures d'ancrage. La simplification porte sur l'hypothèse selon laquelle une attache dépliée par un choc résistera comme une attache plane. Cette situation n'apparaît pas dans la réalité puisque l'attache est pliée avant d'être sollicitée en traction mais elle permet de qualifier 
quelques formes possibles de pièces, différentes de celle actuellement fabriquée. La modélisation de la fixation de l'attache reste encore éloignée du système réel mais cela n'a aucune incidence sur notre problème. C'est surtout la partie proche du trou oblong qui nous intéresse car c'est à ce niveau que les problèmes surviennent lors des chocs.

La distribution des contraintes de von Mises est étudiée à l'aide d'un algorithme de calcul couplant élastoplasticité et endommagement. Les résultats de calcul ont été analysés à l'aide de plans d'expériences numériques et de surfaces de réponses qui permettent non seulement la visualisation des champs de contraintes, mais aussi qui fournit une approximation numérique des fonctions objectives. Les modèles d'approximations polynomiales de ces fonctions sont une aide précieuse pour une optimisation ultérieure des pièces avec des méthodes mathématiques de sensibilité ou de gradients.

\section{Références}

[1] P. Maurice, Les plans d'expériences par la méthode de Taguchi, ISBN 2-7081-2031-X, 1997

[2] R. Unal, Response surface model building and multidisciplinary optimization using D-Optimal Design, Engineering Management Department, Old Dominion University Norfolk, VA23529-1998

[3] R. Coutin, Les aciers à haute limite d'élasticité à dispersoîdes, propriétés de formage, Cetim Informations, 74, février 1982, ISSN 0399-0001, pp. 60-68

[4] A. Mkaddem, R. Bahloul, A. Potiron, H.S.L.A. steel sheet metal characterisation for metal forming processes by using experimental approaches, The 7th International ESAFORM Conference on Material Forming 2004, Apr. 28-30, Trondheim, Norway
[5] A. Mkaddem, Expérimentation et simulation du pliage de tôles H.L.E. Prévision du comportement en service des pièces pliées, Thèse de doctorat, ENSAM Angers, 16 décembre 2003

[6] J.L. Chaboche, Continuous damage mechanics-A tool to describe phenomena before crack initiation, Nucl. Engrg. Des. 64 (1981) 233-247

[7] R. Hambli, Finite element simulation of fine blanking processes using a pressure- dependant damage model, J. Materials Processing Technology 116 (2001) 252-264

[8] J. Lemaitre, A continuous damage mechanics model for ductile fracture, J. Engineering Materials and Technology 107 (1985) 83-89

[9] J. Lemaitre, J.L. Chaboche, Mécanique des matériaux solides, Dunod Paris, ISBN2-04-018618-2, 1988, pp. 343-444

[10] M.A. Criesfield, Non linear finite element analysis of solids and structures, 1, Wiley, 1991

[11] Hibbit, Karlsson and Sorensen, Inc, ABAQUS Theory Manual, USA, 2001, version 6.2

[12] L. Guillaumat, Aide au pré-dimensionnement de structures composites impactées, Mec. Ind. 1 (2000) 235-240

[13] D. Benoist, Notions sur les plans d'expériences, Technip, 1974

[14] J. Alexis, Pratique industrielle de la méthode de Taguchi, Afnor 1995

[15] S.G. Gilmour, R. Mead, A Bayesian design criterion for locating the optimum point on response surface, Statics \& Probability Letters 64 (2003) 235-242

[16] H. Martins Gomesa, A. Miguel Awruchb, Comparison of response surface and neural network with other methods for structural reliability analysis, Structural Safety 26 (2004) 49-67

[17] S. Wolfram, Le logiciel Mathematica, version 4.2

[18] The Optimization Toolbox with MATLAB. MA : The Mathworks, Inc.; June 18, 2002, version 6.2

Retrouvez nos articles sur le site : www.edpsciences.org/meca 